\title{
A probabilistic approach to sand particle crushing in the triaxial test
}

\author{
Y. NAKATA, A. F. L. HYDE, M. HYODO AND H. MURATA (1999). Géotechnique 49, No. 5, 567-583
}

\section{G. R. McDowell. University of Nottingham}

The authors have addressed the role of particle fracture in the behaviour of crushable soils, and have shown that the statistics of fracture of single grains can provide micromechanical insight into observed behaviour in triaxial tests. Their paper relies on the application of Weibull statistics, but this requires a number of assumptions to be made. Shipway \& Hutchings (1993) noted that for single particles compressed between flat platens, as the ratio of the radius $a_{\mathrm{c}}$ of the contact area to the diameter of the particle $d$ increases, the proportion of the sphere under tension decreases. For some brittle materials, Shipway \& Hutchings (1993) noted that the surface stress needed to cause fracture might be lower than the bulk stress to cause fracture, owing to the presence of many surface flaws. For large contact areas, the particle might therefore fail at a critical value of surface stress. However, for materials with a wide distribution of flaws, bulk failure will still dominate if the contact area is sufficiently small. The application of Weibull (1951) statistics assumes bulk failure, and the authors have used this approach to analyse fracture data from irregular soil particles. This is difficult for two main reasons. First, no analytical solution exists for the stress distribution within a spherical particle. Second, the particles are irregular and undergo multiple fracture. These difficulties can be addressed if all loading geometries are taken to be geometrically similar. To apply Weibull to a complex loading geometry requires integration of some function of stress over the volume of the specimen under tension. As stated, as the contact area increases, the stress distribution in the particle changes, which makes the application of Weibull impossible. If, however, the contact area is taken to be negligible (which will be true for weak, brittle grains), then the integration of stress over the particle volume can be performed, and as a result, Weibull can be applied in the way that the authors have described. This is discussed in detail by McDowell \& Amon (under review).

If Weibull statistics can be applied to soil grains, then the Weibull modulus obtained from crushing tests on particles of a given size should be the same as that determined from a plot of average strength or $37 \%$ strength as a function of size. The authors found that the Weibull modulus for feldspar, given as 1.8 in their Fig. 12(b), did not agree with their data for strength as a function of size in Fig. 13. I suspect that this is because they calculate stress $F / d^{2}$ at failure using the initial diameter of the particle. As the authors note, the feldspar particles showed fracturing of asperities before final catastrophic splitting. The stress for the particle at failure should therefore be calculated using the size of the particle when it fails, which may be much smaller than the initial size of the particle.

The authors have tackled the definition of particle failure, and have proposed alternative definitions based on initial asperity fracture and splitting. However, consider a feldspar particle that slowly crumbles under increasing force before splitting. If the current size of the particle is, say, less than half of the initial size, then we might say that the particle has failed: even though there has been no catastrophic splitting, there is less than half of the particle left. It would be wise to measure the force corresponding to the failure of an asperity as a function of the size of that asperity. It might then be possible to decide on a more scientific definition of failure, for example such that failure occurs when the ratio of the size of the broken asperity $a$ to the initial particle diameter $d$ is such that, say $a / d>0 \cdot 3$. This problem is illustrated further by the author's use of two alternative definitions of fracture in single particle crushing tests, but three alternative definitions in triaxial tests. The third type, which they call 'surface abrasion', must relate to the fracture of very small asperities, as shown in their Figs 17(a)(d). But how small is 'very small'? Clearly, a more scientific definition of failure is called for.

The authors note the strong relationship between the amount of particle breakage and the size of an assumed Modified Cam clay yield surface. The assumed shape of the yield surface is of secondary importance: their data in Figs 14 and 18 suggest that aggregates residing on the same 'elastic line' in $e-\log p^{\prime}$ space have the same evolved particle size distribution. This is in agreement with McDowell \& Bolton (1998), who proposed that particle breakage provides the mechanism for the plastic hardening parameter in soil. They proposed a new work equation that for one-dimensional compression permits no plastic dissipation without particle fracture. This is in agreement with the authors' observation that the amount of particle breakage is related to the size of the current yield surface.

The amount of breakage defined using the $B f$ parameter by the authors has been related to the probability of failure of particles according to the definition of failure based on asperity fracture. The authors therefore correlate the percentage by mass of grains in a triaxial sample greater than the initial smallest particle size, with the number of particles surviving asperity fracture. One would not expect such a strong correlation, as is shown in Fig. 20. A $B f$ value of $0 \cdot 1$, for example, does not imply that $10 \%$ of the original number of particles have had asperities broken off. The asperity fracture survival probability could be much less than $0 \cdot 9$, and $B f$ could still be much less than $0 \cdot 1$. Furthermore, the assumed induced characteristic tensile stress in a particle, given by equation (11), approximates to the macroscopic stress. This implies that all particles carry their equal share of the macroscopic stress. Discrete element simulations (e.g. Cundall and Strack, 1979) show that columns of force form to transmit the major principal stress at intervals of two or three particle diameters. When asperities fracture, or particle splitting occurs, there must be some redistribution of stress, so that new columns of force form. It might be anticipated therefore that as the applied major principal effective stress $\sigma_{1}^{\prime}$ increases by some small amount, the maximum stress induced in all particles during this stress increment might be considerably larger than $\sigma_{1}^{\prime}$. Furthermore, at high stresses the largest grains are protected by the many smaller neighbours that were produced by fracture, and the tensile stresses in the largest grains are much reduced. It would therefore seem that the assumption that all particles have an induced tensile stress approximately equal to the major principal stress is too simple. It should be noted that for the $9.81 \mathrm{MPa}$ isotropic test, the 4.91 MPa shear test, and the $2.94 \mathrm{MPa}$ shear test, the major principal stress (and hence the assumed induced tensile stress within each grain) is approximately the same at the end of all three tests. However, the $B f$ values and final voids ratios are similar only for the $9.81 \mathrm{MPa}$ isotropic test and the $4.91 \mathrm{MPa}$ shear test. For the $2.94 \mathrm{MPa}$ shear test more particle breakage has occurred, and the final voids ratio is lower. As stated previously, breakage is best correlated with amount of plastic hardening: that is, plastic reduction voids ratio, and the size of the yield surface in stress space. The breakage factor may therefore be only loosely correlated with Weibull survival probabilities based on major principal stress.

It seems, therefore, that Weibull statistics offers a useful 
means of gaining micromechanical insight into the behaviour of crushable soils under triaxial conditions. However, it seems that the best breakage parameter is a simple one extracted from particle-grading curves, such as the one the authors propose, and that this breakage parameter correlates best not with Weibull probabilities that require assumptions to be made regarding the induced tensile stresses within the grains. The best correlation is between the breakage factor and the amount of plastic hardening of the aggregate, as measured by the irrecoverable reduction in voids ratio, or the size of the yield surface, which the authors show in Fig. 22. It seems that plastic hardening and particle breakage are inextricably linked.

\section{Authors' reply}

The authors would like to thank McDowell for his constructive discussion. We feel that his comments form a useful and complementary commentary on the paper. Following the pioneering work of McDowell et al. (1996), the authors have applied Weibull (1951) statistics to the crushing of individual particles, and have attempted to establish a relationship between this and the behaviour of particles in triaxial loading. In particular we wanted to examine whether the stress dependence of the mechanical properties of soil is related in some way to microscopic particle crushing. The authors believe that in the medium term soil crushability parameters defined in terms of the properties of individual grains will allow a better description of deformation and strength of soil for design purposes. As a first step towards this we have shown experimentally that the degree of particle crushing can be related to a hardening parameter for the soil such as the size of the yield surface. Indeed, the authors are pleased to note that the empirical relationship between their particle breakage factor and the assumed size of the yield surface is in agreement with the McDowell \& Bolton (1998) work equation for one-dimensional compression.

McDowell in his discussion quite correctly points out the difficulties inherent in the authors' application of Weibull statistics to real soils. In a soil matrix, soil particles will have varying contact areas and potentially more than two points of contact. A given soil particle will be surrounded by many others with different contact conditions. Among these particle contacts some but by no means all of those undergoing crushing will have just two points of contact. Similarly in the case of particles crushed between plates some but not all particles had just two points of contact. The contact area and number of contact points will have depended on the particle shape. Thus it could be argued that the single particle crushing tests using plate loading were to some extent representative of real soil grain contacts.

He refers to the work of Shipway \& Hutchings (1993), who noted that the surface stress needed to cause fracture might be lower than the bulk stress assumed in the application of Weibull (1951). In the case of the quartz particles, the authors do not believe there was a predominance of surface flaws, which would cause surface stress to predominate, although high-resolution microscopy would be required to confirm this. In the case of the weak and brittle feldspar particles, the contact area was probably very small, perhaps making the assumption of bulk failure reasonable. As mentioned above, the single particle crushing tests, in the same way as real soil grains, will have had varying complexity of contact conditons depending on the particle shape. Moreover, the distance between the platens would not necessarily be the same as the diameter of the tensile failure zone. Thus single particle crushing will not always have resulted in an ideal tensile failure, and the crushing mode will have been statistically dependent on the distribution of grain shapes. The strength properties from the plate loading tests are not likely to follow a Weibull distribution based simply on tensile failure but will incorporate statistical variabilities because of the above factors. Thus, in the case of quartz particles, the single particle strengths followed a Weibull distribution with a measurable size effect but the Weibull modulus was less than might be expected from Ashby \& Jones (1986), owing to variation in particle shape and crushing mode.

The authors agree with McDowell that particles should have a similar shape and loading geometry for the application of Weibull statistics, and made the observation that this was a possible explanation for the lack of a clearly observable size effect in the feldspar data. We also acknowledge that the crumbling mode of failure for feldspar led to additional errors being introduced by using the initial particle diameter when defining the characteristic stress at failure. However, even if the stress at failure had been calculated using the size of the particles at failure, the authors believe other factors would have masked the Weibull size effect.

Despite the above reservations, this does not obviate the use of the Weibull function merely as a general statistical function relating a characteristic soil particle crushing stress to its initial size. By using the initial size, the authors were at least able to begin investigating the relationship between single and collective particle crushing. In a matrix, the geometry of the crushed zones or particles cannot be determined and therefore the use of the initial size of a particle was as good a measure as any other.

McDowell suggests the measurement of the force corresponding to the failure of an asperity as a function of the size of that asperity and the use of a more scientific definition of failure in terms of the ratio of the size of the broken asperity to the initial particle diameter. It is clear that the stress corresponding to the failure of an asperity should be measured as a function of the size of the asperity. However, this poses its own problems such as that of measuring the size of the broken asperity and the force acting on the broken area. In addition, during plate loading several asperities might break off at the same time. Clearly, the problem of the application of Weibull statistics to particles of real soil remains to be resolved.

We agree with McDowell's observations on the data presented in our Fig. 20. we accept his argument that the relation between the breakage factor $B_{\mathrm{f}}$ and probability of survival could not to be true for asperity fracture; however, it could be true for particle splitting as the initial size for the soil is more uniform. Unless single particle crushing can be related to macroscopic soil behaviour in a simple and practical way it will be of little interest to most geotechnical engineers. Perhaps as a first step, further clarification is needed of the relationship between a breakage factor and probabilities of survival for individual particles and varying degrees of damage.

McDowell questions our assumptions regarding the transfer of the macroscopic stress on a matrix to individual particles. While we agree with him that discrete element simulations such as that of Cundall \& Strack (1979) provide potentially useful data to resolve this issue, it is still not possible to apply these solutions directly to the calculation of the characteristic crushing stress of an irregular soil particle embedded in a soil matrix, and therefore the authors made the judgement that at this stage using their relatively simple approach was best. Further thoughts on the characteristic crushing stress in a soil matrix will be discussed in Nakata et al. (2001).

\section{REFERENCES}

Ashby, M. F. and Jones, D. R. H. (1986). Engineering materials 2: An introduction to microstructures, processing and design. Oxford: Pergamon Press.

Cundall, P A. and Strack, O. D. L. (1979). A discrete numerical model for granular assemblies. Géotechnique 29, No. 1, 47-65.

McDowell, G. R. and Amon, A. (2000). The application of Weibull statistics to the fracture of soil particles. Soils Found., 40, No. 5, pp 133-141.

McDowell, G. R. and Bolton, M. D. (1998). On the micro mechanics of crushable aggregates. Géotechnique 48, No. 5, 667-679.

McDowell, G. R., Bolton, M. D. and Robertson, D. (1996). The fractal crushing of granular materials. J. Mech. Phys. Solids 44, No. 12, 2079-2102. 
Nakata, Y., Kato, Y., Hyodo, M., Hyde, A. F. L. and Murata, H. (2001). One-dimensional compression behaviour of uniform sand related to single particle crushing strength. Soils Found., 41, No. 2.

Shipway, P. H. and Hutchings, I. M. (1993). Fracture of brittle spheres under compression and impact loading. I. Elastic stress distributions Phil. Mag. A 67, No. 6, 1389-1404.

Weibull, W. (1951). A statistical distribution function of wide applicability. J. Appl. Mech. 18, 293-297. 